\title{
German Registry for Cardiac Operations and Interventions in Patients with Congenital Heart Disease: Report 2020-Comprehensive Data from 6 Years of Experience
}

\author{
Andreas Beckmann ${ }^{1}$ S. Dittrich 20 \\ U. M. M. Bauer ${ }^{8}$ M. Hofbeck ${ }^{9}$ \\ C. Arenz ${ }^{3} \quad$ O.N. Krogmann 4 \\ A. Horke 5 \\ A. Tengler 6 \\ R. Meyer ${ }^{7}$
}

${ }^{1}$ German Society for Thoracic and Cardiovascular Surgery, Langenbeck-Virchow-Haus, Berlin, Germany

2 Pediatric Cardiology Department, University of Erlangen, Erlangen, Germany

${ }^{3}$ German Pediatric Heart Center, University of Bonn, Bonn, Germany

Address for correspondence Andreas Beckmann, MD, Deutsche Gesellschaft für, Thorax-, Herz- und Gefäßchirurgie [DGTHG], Langenbeck-Virchow-Haus, Luisenstr. 58-59, 10117 Berlin, Germany

${ }^{4}$ Clinic for Pediatric Cardiology-Congenital Heart Defects, Heart

Center Duisburg, Duisburg, Germany

${ }^{5}$ Division of Congenital Heart Surgery, Hannover Medical School, Hannover, Germany

${ }^{6}$ Division of Pediatric Cardiology and Pediatric Intensive Care; Ludwig Maximilian University of Munich, Munich, Germany

${ }^{7}$ BQS Institute for Quality and Patient Safety, Hamburg, Germany

8 National Register for Congenital Heart Defects, Berlin, Germany

${ }^{9}$ Department of Pediatric Cardiology, Pulmology and Intensive Care, University Children's Hospital, Tübingen, Germany

\author{
Abstract \\ Keywords \\ - congenital heart \\ disease \\ - outcomes \\ - multicenter registry \\ - pediatric cardiology \\ - congenital heart \\ surgery
}

Background Based on a quality assurance initiative of the German Society for Thoracic and Cardiovascular Surgery (DGTHG) and the German Society for Pediatric Cardiology and Congenital Heart Defects (DGPK), a voluntary registry was founded for assessment of treatment and outcomes of patients with congenital heart disease in Germany. This evaluation by the German Registry for cardiac operations and interventions in patients with congenital heart disease reports the data and the outcome over a 6-year period in patients undergoing invasive treatment.

Methods This real-world database collects clinical characteristics, in-hospital complications, and medium-term outcome of patients who underwent cardiac surgical and interventional procedures within the prospective, all-comers registry. Patients were followed-up for up to 90 days.

Results In the period from 2013 to 2018 , a total of 35,730 patients, 39,875 cases, respectively 46,700 procedures were included at up to 31 German institutions. The cases could be subcategorized according to the treatment intention into 21,027 (52.7\%) isolated operations, 17,259 (43.3\%) isolated interventions, and 1,589 (4.0\%) received

December 10, 2020

accepted

December 11, 2020
DOI https://doi.org/ 10.1055/s-0041-1722978. ISSN 0171-6425.

\footnotetext{
(C) 2021. The Author(s).

This is an open access article published by Thieme under the terms of the Creative Commons Attribution-NonDerivative-NonCommercial-License, permitting copying and reproduction so long as the original work is given appropriate credit. Contents may not be used for commercial purposes, or adapted, remixed, transformed or built upon. (https://creativecommons.org/ licenses/by-nc-nd/4.0/) Georg Thieme Verlag KG, Rüdigerstraße 14, 70469 Stuttgart, Germany
} 
with multiple procedures. Of these, 4,708 (11.8\%) were performed in neonates, 10,047 (25.2\%) in infants, $19,351(48.5 \%)$ in children of 1 to 18 years, and 5,769 (14.5\%) in adults. Also, 15,845 (33.9\%) cases could be allocated to so-called index procedures which underwent a more detailed evaluation to enable meaningful comparability. The mean unadjusted in-hospital mortality of all cases in our registry ranged from $0.3 \%$ in patients with isolated interventions and $2.0 \%$ in patients with surgical procedures up to $9.1 \%$ in patients undergoing multiple procedures.

Conclusion This annually updated registry of both scientific societies represents voluntary public reporting by accumulating actual information for surgical and interventional procedures in patients with congenital heart disease (CHD) in Germany. It describes advancements in cardiac medicine and is a basis for internal and external quality assurance for all participating institutions. In addition, the registry demonstrates that in Germany, both interventional and surgical procedures for treatment of CHD are offered with high medical quality.

\section{Introduction}

During the last decades, major progress has been made in the treatment of patients with congenital heart disease (CHD). This is based on continuous improvements in diagnostic assessment, surgical and interventional procedures, as well as periprocedural intensive care. However, many concepts of treatment are complex and carry a significant risk of morbidity and mortality. Therefore, several registries have been established worldwide to assess the outcome and to improve the quality of various forms of treatment in patients with CHD. ${ }^{1}$ In Germany, at the beginning of this century, cardiac surgical procedures in CHD were subject to a nationwide legally binding external quality assurance. In 2004, this obligatory quality assurance measure, according to $\S 136 \mathrm{ff}$ Social Code Book V, was suspended by the political representatives (Bundeskuratorium) for various reasons. Since quality of care has a major impact on long-term outcome and quality of life of patients, especially those with CHD, the scientific professional societies, German Society for Thoracic and Cardiovascular Surgery (DGTHG) and German Society for Pediatric Cardiology and Congenital Heart Defects (DGPK), took the initiative and the responsibility to initiate a multicenter registry study concerning interventional and surgical therapies in patients with CHD. Following the complex project engineering, setting up of a relational database system and completion of a pilot phase, the nationwide registry started in 2012. The purpose of this report is to describe the structure and to present data from the first 6 years of data entry.

\section{Registry Structure}

The voluntary German Registry for Quality Assurance in CHD (Nationale Qualitätssicherung Angeborene Herzfehler) is approved by the Charité's Ethics Committee (code number: EA2/011/11) and designed to collect data on the treatment and outcomes of patients suffering from CHD in Germany. It is conceived as a prospective, controlled, multicenter regis- try which enables data acquisition focused on interventional and surgical procedures. The executive boards of the societies DGPK and DGTHG addressed all German heart centers performing interventional or surgical procedures for CHD to include patients consecutively. Inclusion criteria are the presence of any congenital heart defect and the requirement of cardiac surgery or catheter-based intervention. The decisive factor for inclusion is the intention to treat the procedure, not its success. Since treatment of CHD extends into adulthood, inclusion of patients in this registry is not limited to a specific age. According to European Union's General Data Protection Regulation (EU-GDPR), participation in this voluntary registry requires informed consent either of the patient or guardian. Hence the only exclusion criterion for participation is the absence of written informed consent.

Each included patient received a unique personal identification (PID), valid for life, which generates a pseudonym. Based on the PID, any invasive interventional or cardiac surgical procedure can be assigned exactly to each individual patient, even if the treatment is provided in different institutions. This enables longitudinal, procedure-related data acquisition, as well as short-, mid- and long-term evaluations.

\section{Materials and Methods}

After checking for inclusion and exclusion criteria, the data are entered into a web-based electronic case record form (eCRF) by the participating institution. The eCRF is coupled with a patient identifier (PID) generator, ${ }^{2}$ which serves as central identity management. By dividing the individual patient-related data into hard and soft criteria and phonetic algorithms, the unambiguity of a patient's data upon pseudonymization is guaranteed.

The eCRF consists of three parts including a general, a therapy related, and a follow-up sheet. In the general, sheet structured information on the patient's medical history is collected and can be updated over the entire treatment course, even at distinguished time marks and also in different institutions. 
The therapy-related sheet consists of three subsheets: admission, procedures (surgery, intervention, or hybrid procedure), and discharge. The treatment of a patient during one hospital admission is defined as a case. The structure of the database provides the possibility to record more than one procedure if the patient requires subsequent surgical, interventional, or any combination of procedures during one admission. Coding of the procedures is based on the International Pediatric and Congenital Cardiac Code. ${ }^{3}$ If a surgical or interventional procedure contains separate components, the most complex one is defined as the main procedure and forms the basis of subgrouping. The follow-up sheet records the patient's state of health 90 days after the initial procedure (follow-up). For every readmission, a new patient-related case with separate therapy-related sheets is created for documentation of further surgical or interventional procedures.

The database uses the ixserv-software (ixmid Software Technologie GmbH, Köln, Germany) which enables plausibility and completeness checks during documentation, as well as query management. For data security and protection, the IT platform of the National Register for Congenital Heart Defects is used (registration no.: 531.390, Berlin Commissioner for Data Protection and Freedom of Information). After completion of data records, a central online monitoring is performed, followed by an export for descriptive analyses and statistical evaluations. Risk adjustment models for operations and interventions were implemented in 2014. The risk group classification for operations is performed according to the internationally recognized society of thoracic surgeons-european association for cardio-thoracic surgery (STAT) mortality score ${ }^{4,5}$ while risk group adjustment for interventions is based on the internationally acknowledged Bergersen's score. ${ }^{6}$ These classifications cover five categories for surgical and four for interventional procedures. However, it has to be remembered that the two risk scores are not comparable, as they are based on different morbidity expectations and different evaluations of special features and complications.

In addition to procedure-related data, various indicators, including the occurrence of adverse events, in-hospital mortality, and 30-day and 90-day mortality, are evaluated. Major and minor adverse events following surgical procedures are classified according to the society of thoracic surgeons (STS) morbidity classification. ${ }^{7}$ Adverse events following interventional procedures are grouped into five categories according to the classification of Bergersen. ${ }^{6}$

In the evaluation of different procedure types, the national quality assurance discriminates cases with isolated interventions, isolated operations, and multiple and hybrid procedures. Cases referring to isolated interventions and operations include all hospital admissions with intention to treat the patient with a single procedure and those who require subsequent treatment of a complication by a second procedure during the same admission. From 2015, cases with multiple procedures include patients who underwent more than one procedure during one admission based on a predefined concept (e.g., arterial switch operation following balloon atrioseptostomy). Prior to that, these cases were assigned to surgical or interventional cases. Hybrid proce- dures are defined as a treatment combining surgical and interventional elements during the same procedure.

To allow a more detailed and differentiated view on specific procedures in homogeneous subgroups of CHD, 15 index (nine surgical and six interventional) procedures were defined. The surgical procedures include treatment of atrial septal defect (ASD), ventricular septal defect (VSD), atrioventricular septal defect (AVSD), aortic coarctation, transposition of the great arteries with intact ventricular septum, the tetralogy of Fallot, total cavopulmonary connection, hypoplastic left heart syndrome, and pulmonary valve replacement. Interventions include ASD and VSD closure, treatment of aortic coarctation and recoarctation, closure of patent ductus arteriosus, and percutaneous pulmonary valve replacement.

Each year, the responsible scientific societies carry out an annual report based on two types of analyses. The national report summarizes aggregated and anonymized results of all participating institutions, compared with the previous year. In addition, separate institution-related reports contain the analyses of each participating heart center compared with the national results. Due to contractual arrangements, the institutional report is confidential and not intended for public access. Upon request, each institution can receive an electronic copy of its evaluated data for further use (e.g., transfer to the ECHSA-database).

The structure of the registry, data acquisition, and evaluation is in accordance with the guidelines of "Good Epidemiological Practice (GEP),"8, "Good Hospital Practice (GCP),"9 and the Declaration of Helsinki for medical research involving human subjects. ${ }^{10}$

\section{Registry Data 2013-2018}

Data and results presented in this report capture assorted data of the years 2013 to 2018 collected from 20 to 24 departments performing surgical and from 24 to 30 departments carrying out interventional procedures (-Fig. 1). During this time period, a total number of 35,730 patients was included, leading to 39,875 cases, and resp. 46,700 procedures (-Fig. 2 ). The cases could be subdivided into 17,259 interventional, resp. 21,027 surgical cases, and 1,589 with multiple procedures (-Fig. 3). Since so-called hybrid procedures represented a less than rare therapy option accounting for less than $1 \%$ of the cases, they were grouped under multiple procedures. Overall, 4,708 (11.8\%) of all cases were performed in neonates, 10,047 (25.2\%) in infants, $19,351(48.5 \%)$ in children/adolescents at age of 1 to 18 years, and 5,769 (14.5\%) in adults (- Fig. 4). Also, $3,085(17.9 \%)$ of the 17,259 isolated interventional procedures and 2,612 (12.4\%) of 21,027 isolated surgical procedures were performed in adults. About $45 \%$ of the surgical procedures were performed in the neonatal period or infancy, while $60 \%$ of the interventional procedures were recorded in children/adolescents between 1 and 18 years of age. Corresponding to the known predominance of the male gender in congenital heart defects, more procedures were performed in male patients.

Risk adjustment was implemented in 2014, therefore evaluation of data based on risk adjustment is limited to the years 2014 to 2018. Since not all of the surgical and 
interventional procedures could be assigned to specific risk groups of the STAT mortality score and the Bergersen score, $8.2 \%$ of the surgical and $11.3 \%$ of interventional cases during the observed period are not classified according to these complexity scores (-Table 1). Among the classified cases, approximately $50 \%$ of the interventional cases were in group 2 and approximately $44 \%$ of the surgical cases in group 1 (-Fig. 5A, B). Approximately $16 \%$ of all surgical cases were found in the highest risk categories 4 and 5 of the STAT mortality score, and almost $12 \%$ of the interventional cases were in risk category 4 of the Bergersen score. Newborns and infants were disproportionately overrepresented in these high-risk categories, reflecting the need for early treatment of complex congenital heart malformations in these age groups (-Figs. 6 and 7).

The results for the entire cohort during the survey years showed a documented in-hospital mortality of 0.2 to $0.8 \%$ for isolated interventions and 1.4 to $2.6 \%$ for isolated operations. The observed mortality rate was highest for cases requiring multiple procedures with a range of 6.2 to $10.4 \%$ (-Table 2). This can be explained by the fact that in the majority of cases, the combination of more than one surgical and interventional procedure was required especially in newborns and infants with complex heart malformations, representing the highest risk groups. In accordance with the clearly different treatment characteristics for isolated interventions, isolated operations, and multiple procedures, we observed differences not only in the mortality but also in the incidence of observed adverse events (-Fig. 8). Additionally, there were significant differences regarding mechanical ventilation, duration of intensive care treatment, and hospital stay (-Fig. 9). The analysis of in-hospital mortality by age group showed the highest level in newborns (mean 6.7\%) and the lowest in children/adolescents 1 to 18 years of age (mean, $0.5 \%$; - Table 3 ). In addition, there were no significant variations of mortality rates during the span of the survey period (-Table 3). Also, 30- and 90-day mortalities were quite constant ( - Table 4 ).

During the study period, a total of 17,259 isolated interventions was recorded in the registry. In $52.3 \%$ of these patients, an invasive treatment had already been performed during preceding hospital admissions. The vast majority of isolated interventions was performed without adverse events (range: $83.5-94.5 \%$; - Fig. 8). Also, $8.7 \%(n=87)$ of the observed adverse events fell into the categories "major" or "catastrophic."

From 2013 to 2018, 21,027 cases were categorized as isolated operations leading to 24,537 operative procedures. In almost two-thirds of these cases, complex surgical procedures were required, addressing more than one congenital anomaly. The specific surgery process indicators showed that $18,157(74.0 \%)$ of the surgical procedures were performed under extracorporeal circulation (ECC). The median values of the operation, extracorporeal circulation, and aortic crossclamping times showed no significant changes over the study period. Circulatory arrest was required in 1,282 operations (5.2\%), and selective brain perfusion was used in $2.2 \%$.
Cerebral perfusion was monitored by near-infrared spectroscopy (NIRS) in almost $65.5 \%(n=16,071)$. In $7.2 \%$ of the cases, a second procedure in terms of adverse event management was necessary. Regarding the general process indicators, it should be noted that almost all patients were operated under general anesthesia and needed intensive care therapy postoperatively. The overall mortality of all surgical cases was $2.5 \%$. According to the STAT mortality score, a clear association could be observed between complexity of the procedure and in-hospital mortality (-Fig. 10).

The index procedure groups included a total of 15,845 cases, comprising 9,465 surgical (nine subgroups) and 6,380 interventional cases (six subgroups; -Fig. 11A, B). Interventional ASD closure was the most frequently performed index intervention with several 3,027 (47.4\%), followed by PDA-closure with 1,716 (26.9\%; -Fig. 11A). For the index operations, VSD closure was the most frequent procedure accounting for 1,984 cases (21.0\%), followed by 1,562 (16.5\%) surgical ASD corrections (-Fig. 11B). There was almost zero mortality regarding the interventional index procedures (except for single cases of VSD closure and treatment of recoarctation) and very low mortality concerning the surgical index procedures. Significant changes of in-hospital mortality could not be observed for the different index procedures over the time span from 2013 to 2018 (-Table 5).

\section{Discussion}

This multicenter registry was initiated by the scientific associations DGTHG and DGPK following suspension of the external obligatory quality assurance for surgery of CHD with the intention to overcome two major deficits of the preceding project. First, since surgical and interventional treatment of CHD can no longer be considered as separate entities but rather represent complementary parts of common treatment concepts, the structure of the registry was designed to include the assessment of both treatment modalities. This is supported by the fact that a significant number of procedures in this registry was either performed during the same or subsequent admissions based on combined treatment concepts. Second, the structure of the registry allows longitudinal assessment of patients and of cohorts. Long-term assessment will become increasingly important in CHD since the quality of treatment frequently does not become apparent within the first months after the procedure.

Our data show that this voluntary registry is able to provide a comprehensive overview over included surgical and interventional procedures performed in patients with $\mathrm{CHD}$. Over the study period, both the number of procedures and the results of outcome indicators showed a relatively constant level. According to the data, covering many years, interventional and surgical procedures in Germany were continuously performed at a high-quality level compared with international surveys. This refers both to the entire cohort and to 15 welldefined homogeneous index procedures which underwent 
separate detailed assessment. It has to be emphasized again that interventions and operations must not be understood as competing, but as complementary therapeutic options in the treatment of congenital heart defects. Even in patients with the same anatomic diagnosis, the morphologic and hemodynamic characteristics, as well as other selection criteria for interventional or surgical procedures, may differ considerably. Therefore, procedural results, process numbers, and event rates are frequently unsuitable for direct comparison between these treatment modalities.

The data obtained from this registry are especially important for various aspects of future developments in health care policy. These include, among others, requirements for future training of medical personnel, especially in the context of the increasing shortage in skilled nursing personnel, and the future provision of health care for the increasing subgroup of adults with CHD. ${ }^{11}$

In the future, further efforts should be made to improve the basic configuration of the registry to enable more detailed and advanced risk-adjusted data-analyses. However, changes of data collection should be well considered not to interfere with longitudinal and, especially, long-term data analysis of the registry. Completeness, validity, and further developments of this registry will depend on continued efforts of the DGTHG and the DGPK in close collaboration with all pediatric cardiac and surgical departments in Germany. This will be of outstanding importance to increase patient safety even further and to ascertain the continued high quality of invasive procedures for the treatment of CHD.

\section{Limitations}

This registry is limited by its all-comers registry design and the voluntary participation of patients. However, in our opinion, it is unlikely that this relative lack of completeness would change data quality in a relevant way.

\section{Definitions for Adverse Event Severity (Bergersen et al)}

1. None: no harm, no change in condition, and may have required monitoring to assess for potential change in condition with no intervention indicated. ${ }^{6}$

2. Minor: transient change in condition, not life-threatening, condition returns to baseline, required monitoring, required minor intervention such as holding a medication, or obtaining laboratory test.

3. Moderate: transient change in condition may be lifethreatening if not treated, condition returns to baseline, required monitoring, required intervention such as reversal agent, additional medication, transfer to the intensive care unit for monitoring, or moderate transcatheter intervention to correct condition.

4. Major: change in condition, life-threatening if not treated, change in condition may be permanent, may have re- quired an intensive care unit admission or emergent readmit to hospital, may have required invasive monitoring, and required interventions such as electrical cardioversion or unanticipated intubation or required major invasive procedures or transcatheter interventions to correct condition.

5. Catastrophic: any death, and emergent surgery, or heartlung bypass support (extracorporeal membrane oxygenation) to prevent death with failure to wean from bypass support.

\section{Definition of Major Complications (Jacobs et al)}

1. Postoperative acute renal failure requiring temporary or permanent dialysis. $^{7}$

2. Postoperative neurologic deficit persisting at discharge.

3. Postoperative atrioventricular block requiring permanent pacemaker.

4. Postoperative mechanical circulatory support.

5. Phrenic nerve injury/paralyzed diaphragm.

6. Unplanned reoperation.

\section{Collaborators}

German Quality Assurance / Competence Network for Congenital Heart Defects Investigators Gunter Kerst, Majed Kanaan, Stefan Ostermayer, Jaime F. Vazquez-Jimenez (Aachen); Stephan Schubert, Kai Thorsten Laser, Eugen Sandica (Bad Oeynhausen); Felix Berger, Oliver Miera, Joachim Photiadis, Bernd Opgen-Rhein (Berlin); Johannes Breuer, Ulrike Herberg, Nicole Müller, Martin Schneider, Boulos Asfour (Bonn); Trong Phi Lê (Bremen); Gleb Tarusinov, Jochen Börgermann, Michael Scheid (Duisburg); Frank Pillekamp, Artur Lichtenberg (Düsseldorf); Wolfgang Wällisch, Robert Cesnjevar(Erlangen); Ulrich Neudorf(Essen); Brigitte Stiller, Christoph Zürn, Fatos Ballazhi, Friedhelm Beyersdorf, Fabian Alexander Kari, Johannes Kroll, Matthias Siepe (Freiburg); Christian Jux, Andreas Böning, Hakan Akintürk (Gießen); Thomas Paul, Matthias Sigler, Ulrich Krause, Tim Attmann, Ingo Kutschka, Theodor Tirilomis (Göttingen); Ralph Grabitz, Ia Zakaraia (Halle); Rainer Kozlik-Feldmann, Carsten Rickers, André Rüffer, Jörg Sachweh, Hermann Reichenspurner (Hamburg); Philipp Beerbaum, Axel Haverich, Dietmar Böthig (Hannover); Matthias Gorenflo, Matthias Karck, Tsvetomir Loukanov (Heidelberg); Hashim AbdulKhaliq, Julianna Olchvary, Hans-Joachim Schäfers (Homburg); Thomas Kriebel (Kaiserslautern); Anselm Uebing, Gunther Fischer, Joachim Thomas Cremer, Jens Scheewe (Kiel); Konrad Brockmeier, Gerardus B. W. E. Bennink, Axel Kröner, Thorsten Wahlers (Köln); Ingo Dähnert, Michael A. Borger, Martin Kostelka (Leipzig); Christoph Kampmann (Mainz); Peter Ewert, Jürgen Hörer, Nikolaus A. Haas (München); Hans-Gerd Kehl, Felix Kleinerüschkamp, Edward Malec, Sven Martens, Helmut Baumgartner Münster); Matthias W. Freund, Oliver Dewald (Oldenburg); Frank Uhlemann, Ioannis Tzanavaros (Stuttgart); Jörg Michel, 
Christian Schlensak (Tübingen); Christian Apitz, Michael Kaestner (Ulm); Andreas Heusch (Wuppertal).

$\begin{array}{ll}\text { Abbreviations } \\ \text { ASD } & \text { Atrial septal defect } \\ \text { AVSD } & \text { Atrioventricular septal defect } \\ \text { CHD } & \text { Congenital heart disease } \\ \text { ECC } & \text { Extracorporeal circulation } \\ \text { NW } & \text { Norwood procedure } \\ \text { PDA } & \text { Patent ductus arteriosus } \\ \text { pCoA } & \text { Primary coarctation } \\ \text { ReCoA } & \text { Re-Coarctation } \\ \text { PVR } & \text { Pulmonary valve replacement } \\ \text { TCPC } & \text { Total cavopulmonary connection } \\ \text { TGA-IVS } & \text { Transposition of the great arteries with intact } \\ & \text { ventricular septum } \\ \text { TOF } & \text { Tetralogy of Fallot } \\ \text { VSD } & \text { Ventricular septal defect }\end{array}$

\section{Funding}

The responsible bodies for the registry are the German Society for Thoracic and Cardiovascular Surgery and the German Society for Pediatric Cardiology and Congenital Heart Defects. The registry receives financial support by all participating institutions and donations from the German Heart Foundation (DHS).

Conflict of Interest

None declared.

\section{Acknowledgments}

On behalf of the German Society for Thoracic and Cardiovascular Surgery (DGTHG) and the German Society for Pediatric Cardiology and Congenital Heart Defects (DGPK), the authors would like to thank the heads of the departments and their teams of all participating heart centers in Germany for their continued cooperation and support to realize this registry.

\section{Figures and Tables}

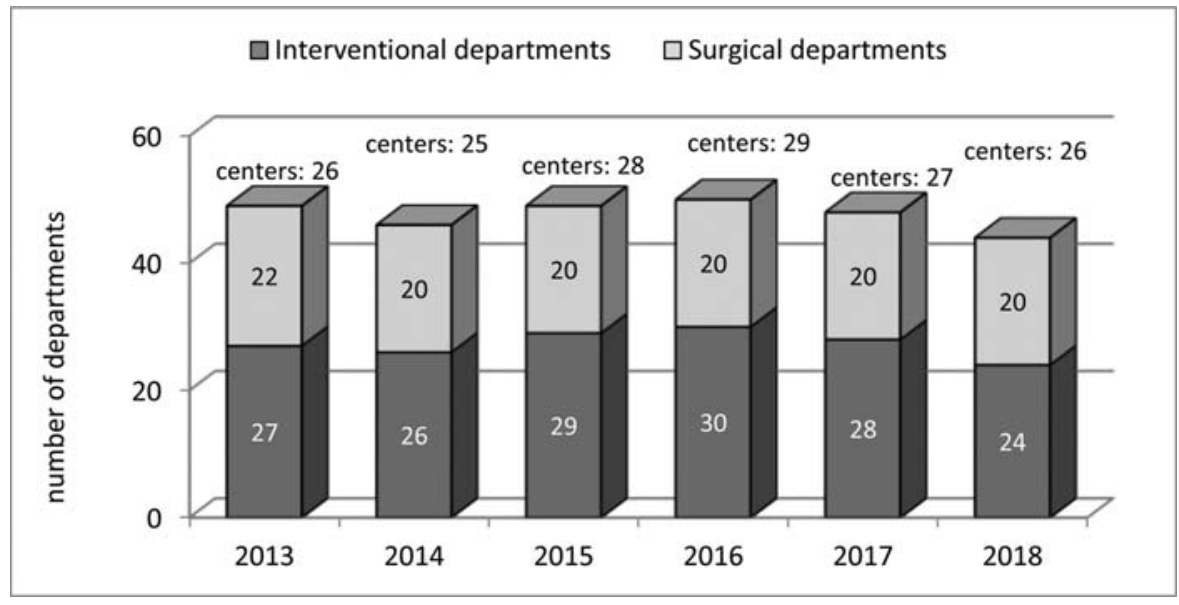

Fig. 1 Participating Institutions 2013 to 2018.

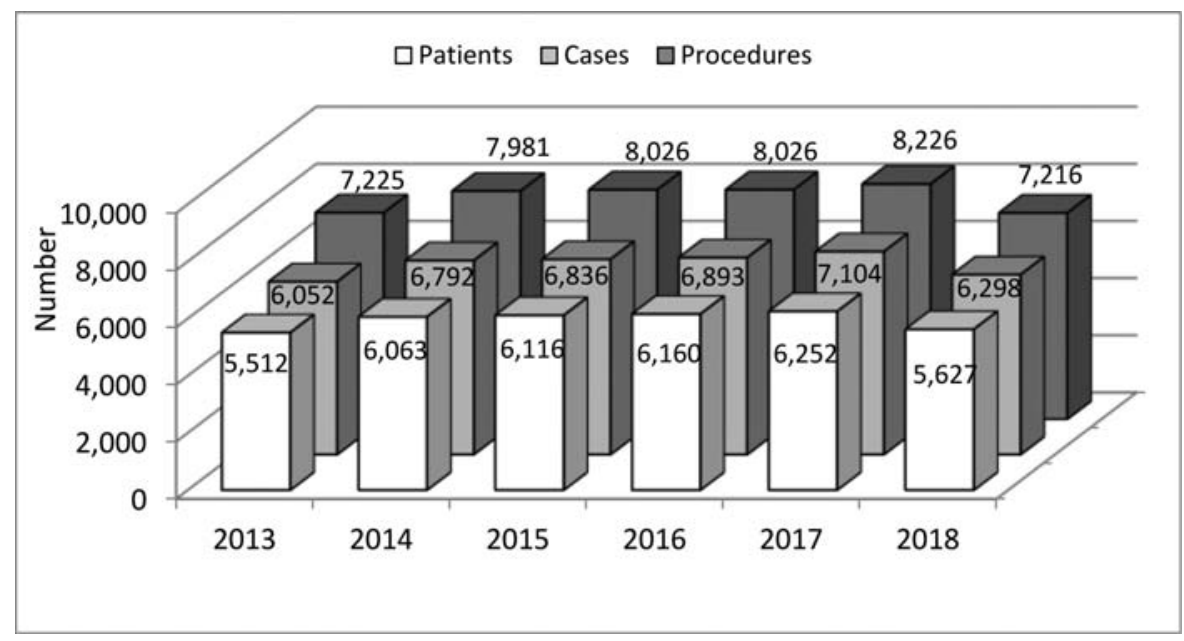

Fig. 2 Patients, cases and procedures 2013 to 2018. 


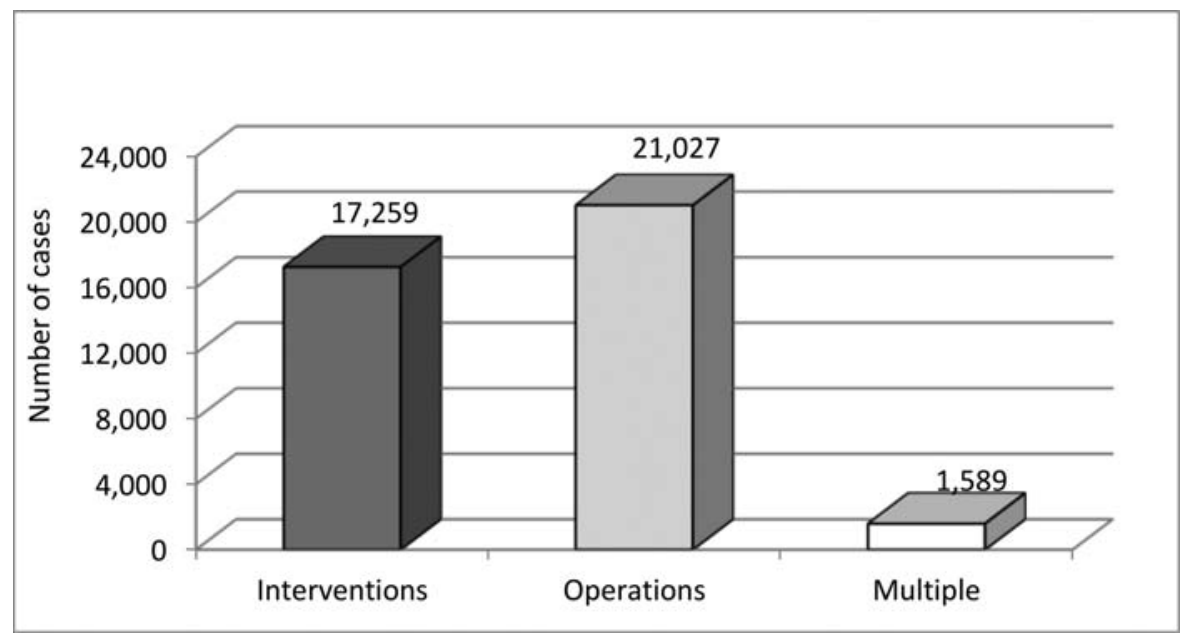

Fig. 3 Categorization by case 2013 to 2018.

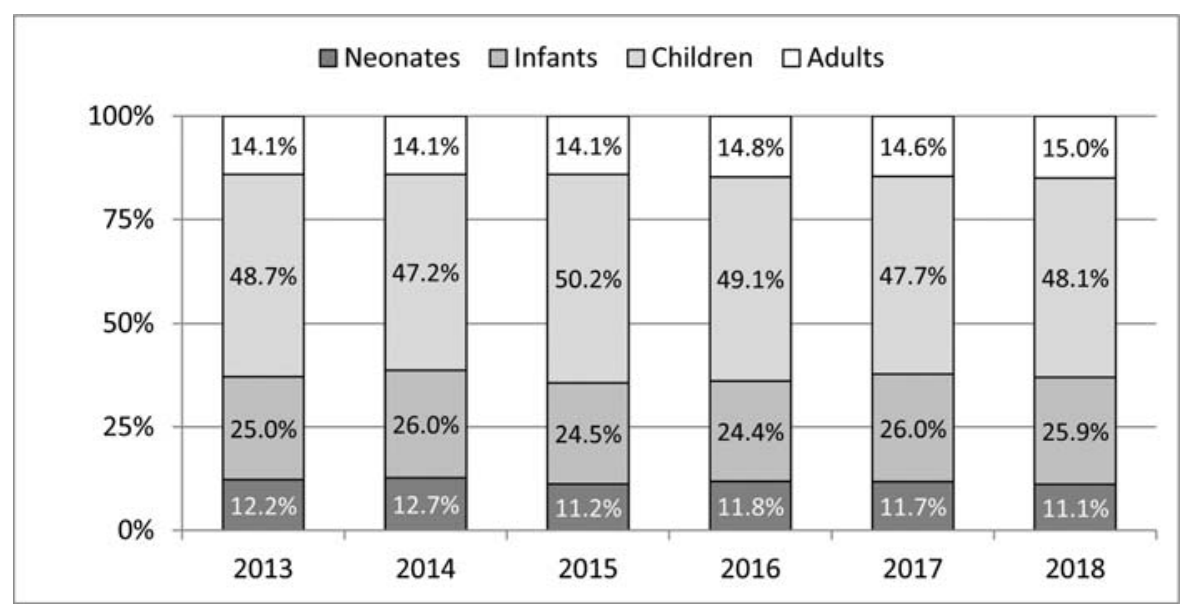

Fig. 4 Age distribution of all cases 2013 to 2018.
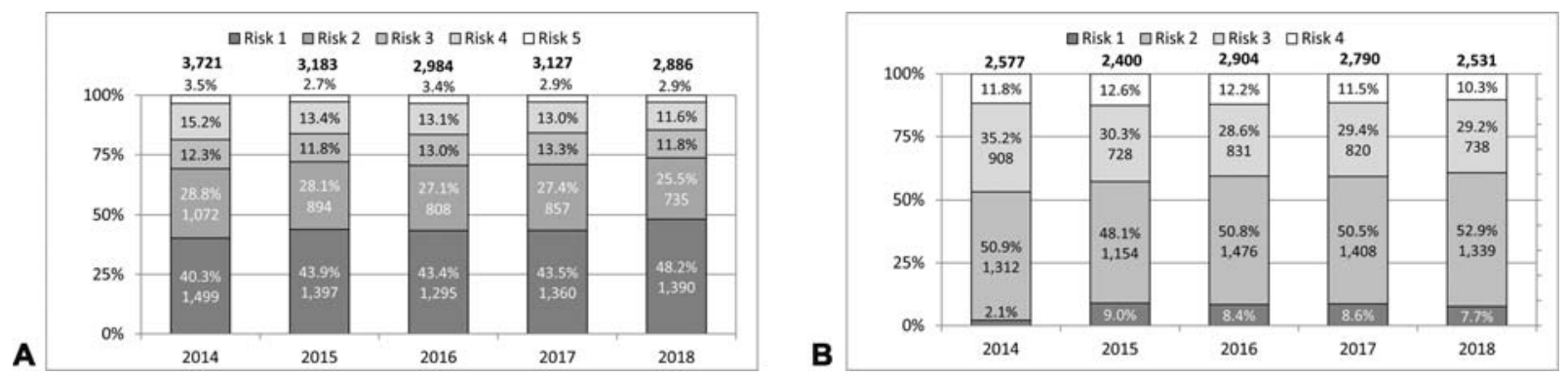

Fig. 5 (A) Surgical cases: risk diversification according to STAT mortality score 2014 to 2018. (B) Interventional cases: risk diversification according to Bergersen's score 2014 to 2018. 
German Registry for Cardiac Operations and Interventions in Patients with Congenital Heart Disease Beckmann

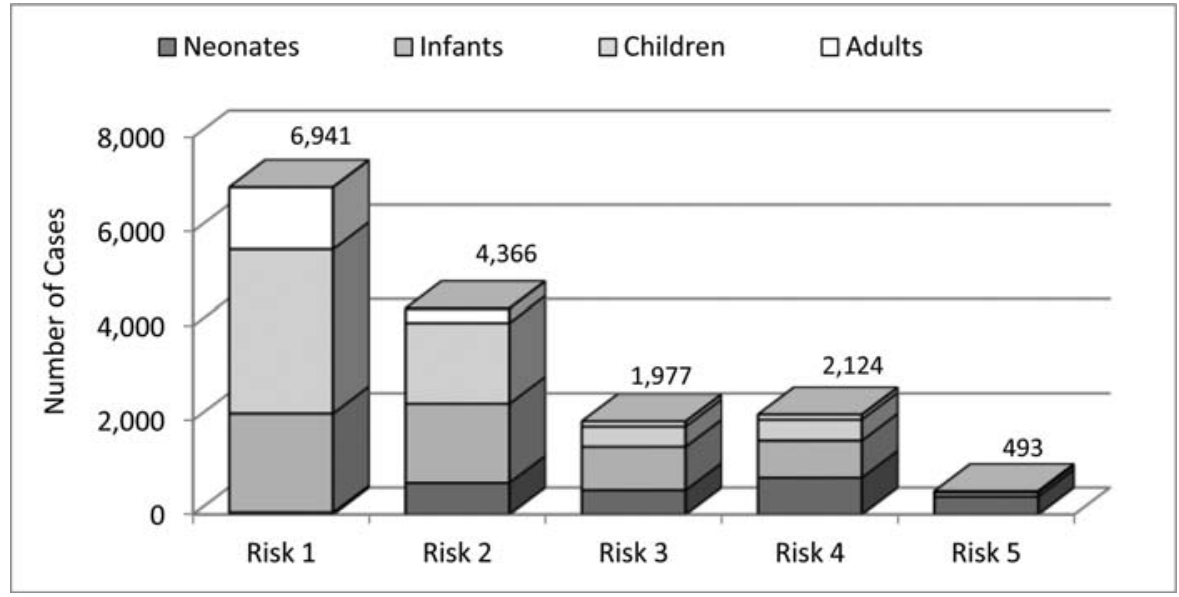

Fig. 6 Surgical cases: STAT mortality categories by age groups 2014 to 2018.

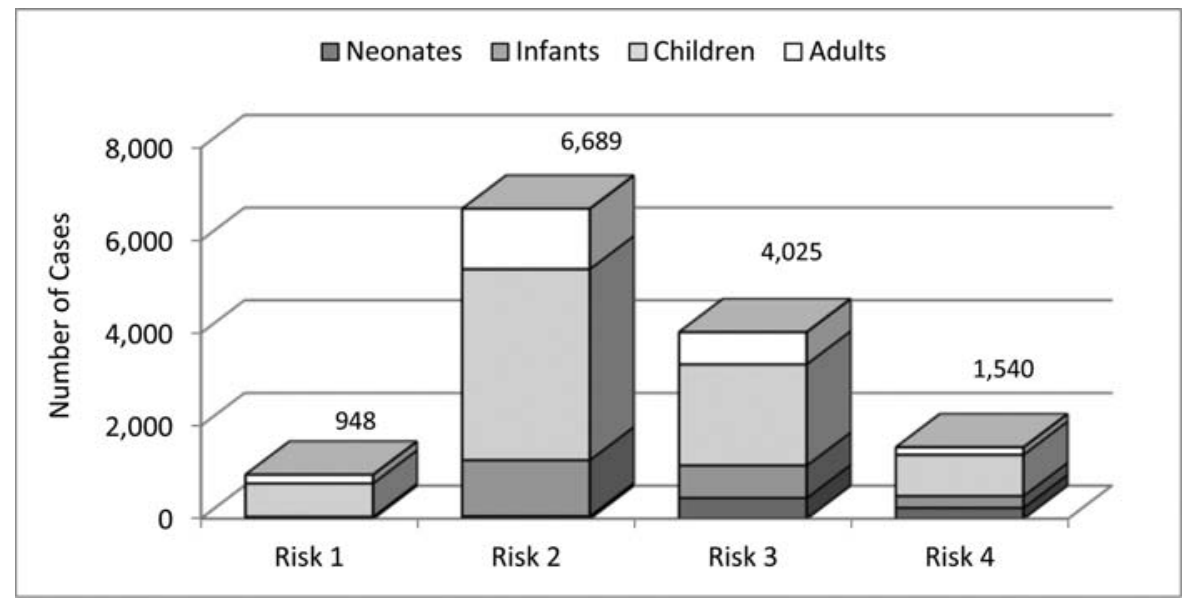

Fig. 7 Interventional cases: Bergersen's score risk categories by age groups 2014 to 2018 .

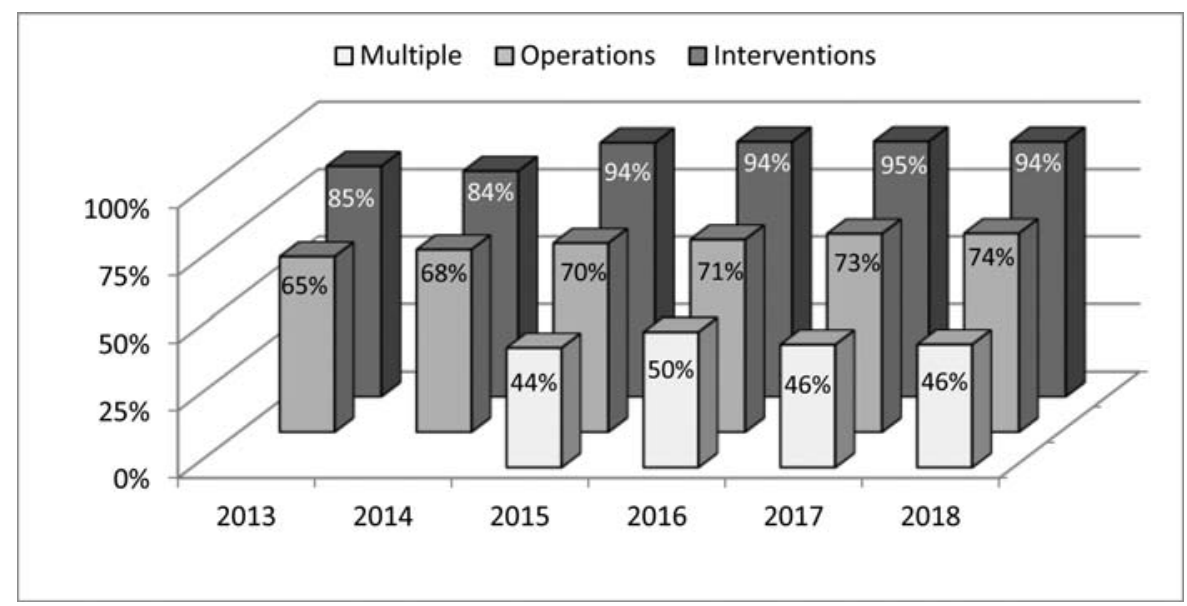

Fig. 8 Cases without adverse events. Note: 2013 to 2014 cases with multiple procedures grouped among operations and interventions. 


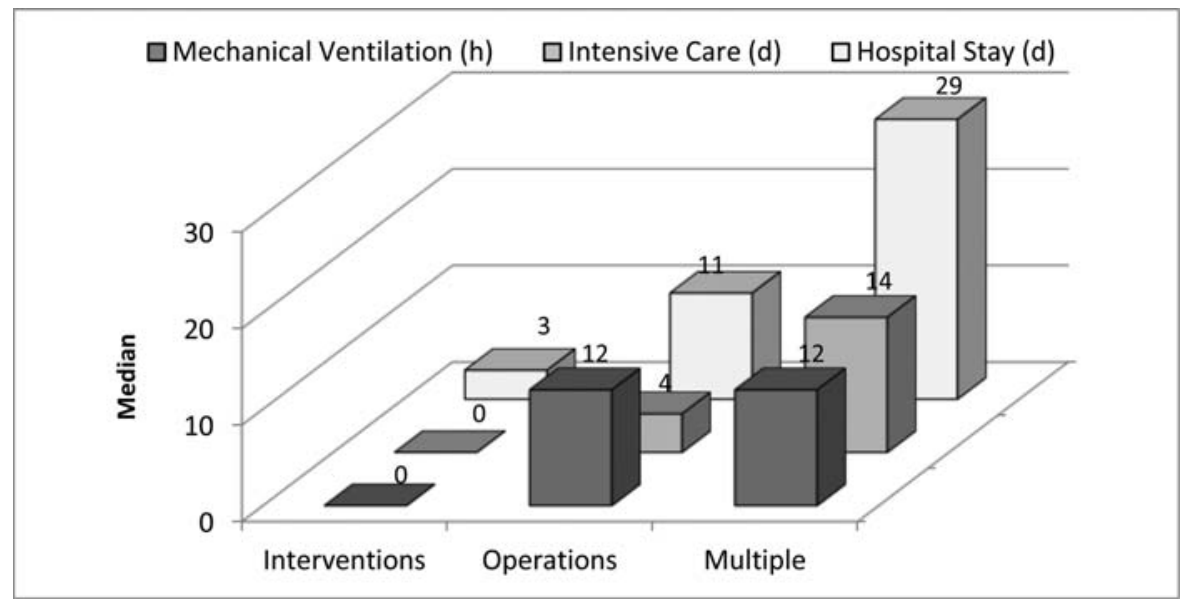

Fig. 9 Process indicators among the different case categories. Note: patients without mechanical ventilation or ICU stay included. ICU, intensive care unit.

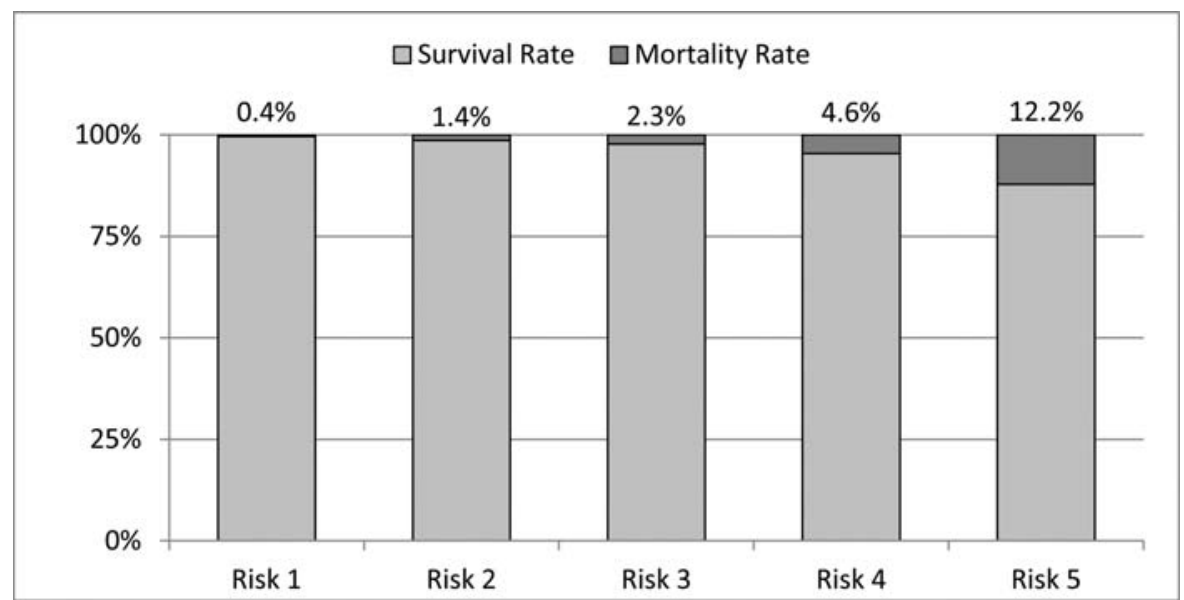

Fig. 10 Surgical cases: association of STAT Mortality Category and in-hospital mortality.
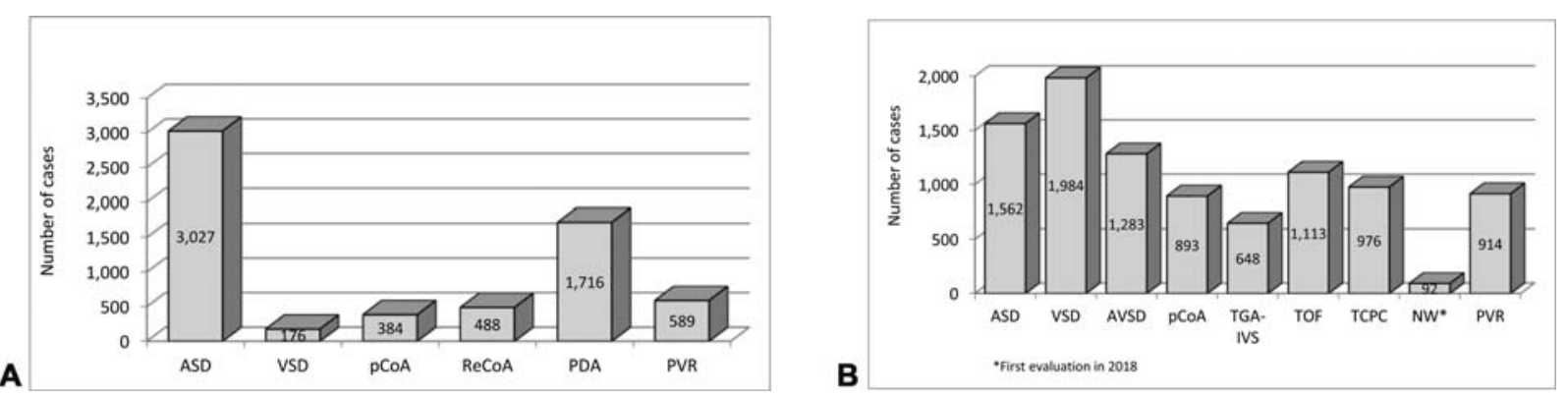

Fig. 11 (A) Index procedures: interventions 2013 to 2018. (B) Index procedures: operations 2013 to 2018. 
Table 1 Applicability of Bergersen's risk score and STAT mortality categories for interventional and surgical cases

\begin{tabular}{|c|c|c|c|c|c|c|c|c|c|c|}
\hline \multirow{4}{*}{$\begin{array}{l}\text { Bergerson Score } \\
\text { Jacobs Score }\end{array}$} & \multirow[b]{2}{*}{ classified } & \multicolumn{2}{|l|}{2014} & \multicolumn{2}{|l|}{2015} & \multicolumn{2}{|l|}{2016} & \multicolumn{2}{|l|}{2017} & \multirow{2}{*}{$\begin{array}{r}2018 \\
\text { not classified }\end{array}$} \\
\hline & & not classified & classified & not classified & classified & not classified & classified & not classified & classified & \\
\hline & $93.8 \%$ & $6.2 \%$ & $82.3 \%$ & $17.7 \%$ & $89.9 \%$ & $10.1 \%$ & $87.9 \%$ & $12.1 \%$ & $90.1 \%$ & $9.9 \%$ \\
\hline & $91.3 \%$ & $8.7 \%$ & $91.6 \%$ & $8.4 \%$ & $92.9 \%$ & $7.1 \%$ & $91.1 \%$ & $8.9 \%$ & $92.5 \%$ & $7.5 \%$ \\
\hline
\end{tabular}

Table 2 In-hospital mortality between different case categories

\begin{tabular}{|l|c|c|c|c|c|c|}
\hline \multirow{3}{*}{$\begin{array}{l}\text { Interventions } \\
\text { Operations }\end{array}$} & $\mathbf{2 0 1 3}$ & $\mathbf{2 0 1 4}$ & $\mathbf{2 0 1 5}$ & $\mathbf{2 0 1 6}$ & $\mathbf{2 0 1 7}$ & $\mathbf{2 0 1 8}$ \\
\cline { 2 - 7 } \\
\cline { 2 - 7 } & $0.8 \%$ & $0.5 \%$ & $0.2 \%$ & $0.2 \%$ & $0.3 \%$ & $0.3 \%$ \\
\cline { 2 - 7 } & $2.6 \%$ & $2.1 \%$ & $1.9 \%$ & $2.5 \%$ & $1.4 \%$ & $1.7 \%$ \\
\hline
\end{tabular}

Table 3 In-hospital mortality (all cases) in different age groups

\begin{tabular}{|c|c|c|c|c|c|c|}
\hline \multirow{5}{*}{$\begin{array}{l}\text { Neonates } \\
\text { Infants } \\
\text { Children } \\
\text { Adults }\end{array}$} & 2013 & 2014 & 2015 & 2016 & 2017 & 2018 \\
\hline & $7.4 \%$ & $5.4 \%$ & $6.9 \%$ & $7.2 \%$ & $6.7 \%$ & $6.6 \%$ \\
\hline & $1.7 \%$ & $1.8 \%$ & $1.4 \%$ & $2.1 \%$ & $1.5 \%$ & $1.7 \%$ \\
\hline & $0.8 \%$ & $0.4 \%$ & $0.3 \%$ & $0.6 \%$ & $0.4 \%$ & $0.5 \%$ \\
\hline & $1.0 \%$ & $0.7 \%$ & $1.1 \%$ & $1.4 \%$ & $0.5 \%$ & $1.0 \%$ \\
\hline
\end{tabular}

Table 4 30/90 days mortality (all cases) 2015 to 2018

\begin{tabular}{|c|c|c|c|c|c|c|c|c|}
\hline \multirow{5}{*}{$\begin{array}{l}\text { Interventions } \\
\text { Operations } \\
\text { Multiple }\end{array}$} & \multicolumn{2}{|c|}{2015} & \multicolumn{2}{|c|}{2016} & \multicolumn{2}{|c|}{2017} & \multicolumn{2}{|c|}{2018} \\
\hline & $30 \mathrm{~d}$ & $90 \mathrm{~d}$ & $30 \mathrm{~d}$ & $90 \mathrm{~d}$ & $30 \mathrm{~d}$ & $90 \mathrm{~d}$ & $30 \mathrm{~d}$ & $90 \mathrm{~d}$ \\
\hline & $0.3 \%$ & $0.6 \%$ & $0.4 \%$ & $1.0 \%$ & $0.4 \%$ & $0.8 \%$ & $0.4 \%$ & $0.8 \%$ \\
\hline & $2.0 \%$ & $2.5 \%$ & $2.8 \%$ & $4.4 \%$ & $1.7 \%$ & $3.1 \%$ & $2.2 \%$ & $3.2 \%$ \\
\hline & $5.6 \%$ & $8.5 \%$ & $9.0 \%$ & $14.3 \%$ & $11.1 \%$ & $15.9 \%$ & $10.4 \%$ & $18.8 \%$ \\
\hline
\end{tabular}

Table 5 Unadjusted in-hospital mortality of surgical index procedure cases

\begin{tabular}{|c|c|c|c|c|c|c|}
\hline & 2013 & 2014 & 2015 & 2016 & 2017 & 2018 \\
\hline ASD & $0.0 \%$ & $0.0 \%$ & $0.0 \%$ & $0.0 \%$ & $0.0 \%$ & $0.0 \%$ \\
\hline VSD & $0.2 \%$ & $0.0 \%$ & $0.0 \%$ & $0.0 \%$ & $0.0 \%$ & $0.0 \%$ \\
\hline AVSD & $1.4 \%$ & $1.8 \%$ & $2.0 \%$ & $0.9 \%$ & $0.0 \%$ & $0.9 \%$ \\
\hline pCoA & $0.0 \%$ & $0.0 \%$ & $0.6 \%$ & $0.7 \%$ & $0.7 \%$ & $0.0 \%$ \\
\hline TGA-IVS & $0.0 \%$ & $0.8 \%$ & $0.0 \%$ & $0.0 \%$ & $1.8 \%$ & $2.2 \%$ \\
\hline TOF & $1.1 \%$ & $0.6 \%$ & $1.0 \%$ & $1.7 \%$ & $0.5 \%$ & $1.2 \%$ \\
\hline TCPC & $2.9 \%$ & $0.0 \%$ & $1.1 \%$ & $1.3 \%$ & $1.1 \%$ & $0.0 \%$ \\
\hline Norwood* & & & & & & $19.6 \%$ \\
\hline PVR & $1.5 \%$ & $0.9 \%$ & $0.0 \%$ & $1.6 \%$ & $0.8 \%$ & $4.7 \%$ \\
\hline
\end{tabular}

\section{References}

1 Vener DF, Gaies M, Jacobs JP, Pasquali SK. Clinical databases and registries in congenital and pediatric cardiac surgery, cardiology, critical care, and anesthesiology worldwide. World J Pediatr Congenit Heart Surg 2017;8(01):77-87

2 Faldum A, Pommerening K. An optimal code for patient identifiers. Comput Methods Programs Biomed 2005;79(01):81-88

3 Franklin RCG, Béland MJ, Colan SD, et al. Nomenclature for congenital and paediatric cardiac disease: the International Paediatric and Congenital Cardiac Code (IPCCC) and the Eleventh
Iteration of the International Classification of Diseases (ICD-11) Cardiol Young 2017;27(10):1872-1938

4 O'Brien SM, Clarke DR, Jacobs JP, et al. An empirically based tool for analyzing mortality associated with congenital heart surgery. J Thorac Cardiovasc Surg 2009;138(05): $1139-1153$

5 Jacobs JP, O'Brien SM, Pasquali SK, et al. Variation in outcomes for risk-stratified pediatric cardiac surgical operations: an analysis of the STS congenital heart surgery database. Ann Thorac Surg 2012; 94(02):564-571, discussion 571-572 
6 Bergersen L, Gauvreau K, Marshall A, et al. Procedure-type risk categories for pediatric and congenital cardiac catheterization. Circ Cardiovasc Interv 2011;4(02):188-194

7 Jacobs ML, O’Brien SM, Jacobs JP, et al. An empirically based tool for analyzing morbidity associated with operations for congenital heart disease. J Thorac Cardiovasc Surg 2013;145(04): 1046-1057.e1

8 Hoffmann W, Latza U, Baumeister SE, et al. Guidelines and recommendations for ensuring good epidemiological practice (GEP): a guideline developed by the German Society for Epidemiology. Eur J Epidemiol 2019;34(03):301-317
9 Guideline for good clinical practice. https://www.ema.europa.eu/en/ich-e6-r2-good-clinical-practice

10 World Medical Association. WMA Declaration of Helsinki - Ethical Principles for Medical Research Involving Human Subjects. Accessed January 11, 2021 at: https://www.wma.net/policies-post/wma-declaration-of-helsinki-ethical-principles-for-medical-research-involving-human-subjects/

11 Kaemmerer H, Bauer U, de Haan F, et al. Recommendations for improving the quality of the interdisciplinary medical care of grown-ups with congenital heart disease (GUCH). Int J Cardiol 2011;150(01):59-64 